Support function method for inverse obstacle scattering problems, In the book "Acoustics, mechanics and related topics of mathematical analysis", World Scientific, New Jersey, 2003, pp.178-184. (ed. A.Wirgin) 


\title{
SUPPORT FUNCTION METHOD FOR INVERSE OBSTACLE SCATTERING PROBLEMS
}

\author{
SEMION GUTMAN \\ Department of Mathematics \\ University of Oklahoma \\ Norman, OK 73019, USA \\ E-mail: sgutman@ou.edu \\ ALEXANDER G. RAMM \\ Department of Mathematics \\ Kansas State University \\ Manhattan, Kansas 66506-2602, USA \\ LMA/CNRS, 31 Chemin Joseph Aiguier \\ Marseille 13402, cedex 20, France \\ E-mail: ramm@math.ksu.edu
}

\begin{abstract}
The knowledge of the Support Function (SF) of a smooth and strictly convex obstacle allows one to reconstruct the obstacle. The SF can be computed if the Scattering Amplitude is known for large values of the wave number $k$. For the values of $k$ in the resonance region, the SF can only be found approximately. Nevertheless, the results of this paper show that such an SF can be used to find an approximate location of the obstacle. The method is inexpensive, and it does not require extensive data.
\end{abstract}

\section{Introduction}

Let an obstacle be a bounded domain $D \subset \mathbb{R}^{2}$ with a Lipschitz boundary $\Gamma$. Fix a frequency $k>0$ and denote the exterior domain by $D^{\prime}=\mathbb{R}^{2} \backslash \bar{D}$. Let $\alpha \in S^{1}$, and the incident field be

$$
u_{0}(\mathbf{x})=e^{i k \mathbf{x} \cdot \alpha}, \quad \mathbf{x} \in D^{\prime} .
$$

The Direct Acoustic Obstacle Scattering Problem for the Dirichlet boundary conditions consists of finding the total field

$$
u(\mathbf{x}, k)=u_{0}(\mathbf{x})+v(\mathbf{x}), \quad \mathbf{x} \in D^{\prime}
$$

such that

$$
\Delta u+k^{2} u=0, \quad \mathbf{x} \in D^{\prime},
$$




$$
u(\mathbf{x})=0, \quad \mathbf{x} \in \Gamma,
$$

and the scattered field $v(\mathbf{x})$ satisfies the Sommerfeld radiation condition

$$
\lim _{|\mathbf{x}| \rightarrow \infty} \sqrt{|\mathbf{x}|}\left(\frac{\partial v}{\partial|\mathbf{x}|}-i k v\right)=0
$$

where the limit is attained uniformly for all the directions $\mathbf{x} /|\mathbf{x}|, \mathbf{x} \in \mathbb{R}^{2}$.

The scattered field $v(\mathbf{x})$ has an asymptotic representation

$$
v(\mathbf{x})=\frac{e^{i k|\mathbf{x}|}}{\sqrt{|\mathbf{x}|}}\left\{A\left(\alpha^{\prime}, \alpha\right)+O\left(\frac{1}{|\mathbf{x}|}\right)\right\}, \quad|\mathbf{x}| \rightarrow \infty,
$$

where the uniquely defined function $A\left(\alpha^{\prime}, \alpha\right)$ is called the Scattering Amplitude of the Obstacle Scattering Problem, see, e.g. ${ }^{2}$.

The Inverse Scattering Problem consists of finding the obstacle $D$ from the Scattering Amplitude, or similarly observed data. In this note the Support Function Method (SFM) originally described in a 3 -D setting in ${ }^{2}$ is used to approximately locate the obstacle $D$. The SFM is described in Section 2, and the numerical results are presented in Section 3.

\section{The Support Function Method (SFM).}

The SFM was originally developed in ${ }^{2}$, pp 94-99. It identifies smooth, strictly convex, obstacles from the knowledge of the Scattering Amplitude at high wave numbers $k$. In this paper we study the numerical performance of the SFM in the resonance region. One can restate the SFM in a 2-D setting as follows.

Let $D \subset \mathbb{R}^{2}$ be a smooth and strictly convex obstacle with the boundary $\Gamma$. Let $\nu(\mathbf{y})$ be the unique outward unit normal vector to $\Gamma$ at $\mathbf{y} \in \Gamma$. Fix an incident direction $\alpha \in S^{1}$. Then the boundary $\Gamma$ can be decomposed into the following two parts:

$$
\Gamma_{+}=\{\mathbf{y} \in \Gamma: \nu(\mathbf{y}) \cdot \alpha<0\}, \text { and } \Gamma_{-}=\{\mathbf{y} \in \Gamma: \nu(\mathbf{y}) \cdot \alpha \geq 0\}
$$

which are, correspondingly, the illuminated and the shadowed parts of the boundary for the chosen incident direction $\alpha$.

Given $\alpha \in S^{1}$, its specular point $\mathbf{s}_{\mathbf{0}}(\alpha) \in \Gamma_{+}$is defined from the condition:

$$
\mathbf{s}_{\mathbf{0}}(\alpha) \cdot \alpha=\min _{\mathbf{s} \in \Gamma_{+}} \mathbf{s} \cdot \alpha
$$

Note that the equation of the tangent line to $\Gamma_{+}$at $\mathbf{s}_{\mathbf{0}}$ is

$$
<x_{1}, x_{2}>\cdot \alpha=\mathbf{s}_{\mathbf{0}}(\alpha) \cdot \alpha,
$$


and

$$
\nu\left(\mathbf{s}_{\mathbf{0}}(\alpha)\right)=-\alpha .
$$

The Support function $d(\alpha)$ is defined by

$$
d(\alpha)=\mathbf{s}_{\mathbf{0}}(\alpha) \cdot \alpha .
$$

Thus $|d(\alpha)|$ is the distance from the origin to the unique tangent line to $\Gamma_{+}$perpendicular to the incident vector $\alpha$. Since the obstacle $D$ is assumed to be convex

$$
D=\cap_{\alpha \in S^{1}}\left\{\mathbf{x} \in \mathbb{R}^{2}: \mathbf{x} \cdot \alpha \geq d(\alpha)\right\} .
$$

The boundary $\Gamma$ of $D$ is smooth, hence so is the Support Function. The knowledge of this function allows one to reconstruct the boundary $\Gamma$ using the following procedure.

Parametrize unit vectors $\mathbf{l} \in S^{1}$ by $\mathbf{l}(t)=(\cos t, \sin t), \quad 0 \leq t<2 \pi$ and define

$$
p(t)=d(\mathbf{l}(t)), \quad 0 \leq t<2 \pi .
$$

Equation (9) and the definition of the Support Function give

$$
x_{1} \cos t+x_{2} \sin t=p(t) .
$$

Since $\Gamma$ is the envelope of its tangent lines, its equation can be found from (14) and

$$
-x_{1} \sin t+x_{2} \cos t=p^{\prime}(t) .
$$

Therefore the parametric equations of the boundary $\Gamma$ are

$$
x_{1}(t)=p(t) \cos t-p^{\prime}(t) \sin t, \quad x_{2}(t)=p(t) \sin t+p^{\prime}(t) \cos t .
$$

So, the question is how to construct the Support function $d(\mathbf{l}), \mathbf{l} \in S^{1}$ from the knowledge of the Scattering Amplitude. In 2-D the Scattering Amplitude is related to the total field $u=u_{0}+v$ by

$$
A\left(\alpha^{\prime}, \alpha\right)=-\frac{e^{i \frac{\pi}{4}}}{\sqrt{8 \pi k}} \int_{\Gamma} \frac{\partial u}{\partial \nu(\mathbf{y})} e^{-i k \alpha^{\prime} \cdot \mathbf{y}} d s(\mathbf{y}) .
$$

In the Kirchhoff (high frequency) approximation one sets

$$
\frac{\partial u}{\partial \nu}=2 \frac{\partial u_{0}}{\partial \nu}
$$

on the illuminated part $\Gamma_{+}$of the boundary $\Gamma$, and

$$
\frac{\partial u}{\partial \nu}=0
$$


on the shadowed part $\Gamma_{-}$. Therefore, in this approximation,

$$
A\left(\alpha^{\prime}, \alpha\right)=-\frac{i k e^{i \frac{\pi}{4}}}{\sqrt{2 \pi k}} \int_{\Gamma_{+}} \alpha \cdot \nu(\mathbf{y}) e^{i k\left(\alpha-\alpha^{\prime}\right) \cdot \mathbf{y}} d s(\mathbf{y}) .
$$

Let $L$ be the length of $\Gamma_{+}$, and $\mathbf{y}=\mathbf{y}(\zeta), 0 \leq \zeta \leq L$ be its arc length parametrization. Then

$$
A\left(\alpha^{\prime}, \alpha\right)=-\frac{i \sqrt{k} e^{i \frac{\pi}{4}}}{\sqrt{2 \pi}} \int_{0}^{L} \alpha \cdot \nu(\mathbf{y}(\zeta)) e^{i k\left(\alpha-\alpha^{\prime}\right) \cdot \mathbf{y}(\zeta)} d \zeta
$$

Let $\zeta_{0} \in[0, L]$ be such that $\mathbf{s}_{0}=\mathbf{y}\left(\zeta_{0}\right)$ is the specular point of the unit vector $\mathbf{l}$, where

$$
\mathbf{l}=\frac{\alpha-\alpha^{\prime}}{\left|\alpha-\alpha^{\prime}\right|}
$$

Then $\nu\left(\mathbf{s}_{0}\right)=-\mathbf{l}$, and $d(\mathbf{l})=\mathbf{y}\left(\zeta_{0}\right) \cdot \mathbf{l}$. Let

$$
\varphi(\zeta)=\left(\alpha-\alpha^{\prime}\right) \cdot \mathbf{y}(\zeta) .
$$

Then $\varphi(\zeta)=\mathbf{l} \cdot \mathbf{y}(\zeta)\left|\alpha-\alpha^{\prime}\right|$. Since $\nu\left(\mathbf{s}_{0}\right)$ and $\mathbf{y}^{\prime}\left(\zeta_{0}\right)$ are orthogonal, one has

$$
\varphi^{\prime}\left(\zeta_{0}\right)=\mathbf{l} \cdot \mathbf{y}^{\prime}\left(\zeta_{0}\right)\left|\alpha-\alpha^{\prime}\right|=0 .
$$

Therefore, due to the strict convexity of $D, \zeta_{0}$ is also the unique nondegenerate stationary point of $\varphi(\zeta)$ on the interval $[0, L]$, that is $\varphi^{\prime}\left(\zeta_{0}\right)=0$, and $\varphi^{\prime \prime}\left(\zeta_{0}\right) \neq 0$.

According to the Stationary Phase method

$$
\begin{aligned}
\int_{0}^{L} f(\zeta) e^{i k \varphi(\zeta)} d \zeta= & f\left(\zeta_{0}\right) \exp \left[i k \varphi\left(\zeta_{0}\right)+\frac{i \pi}{4} \frac{\varphi^{\prime \prime}\left(\zeta_{0}\right)}{\left|\varphi^{\prime \prime}\left(\zeta_{0}\right)\right|}\right] \\
& \sqrt{\frac{2 \pi}{k\left|\varphi^{\prime \prime}\left(\zeta_{0}\right)\right|}}\left[1+O\left(\frac{1}{k}\right)\right],
\end{aligned}
$$

as $k \rightarrow \infty$.

By the definition of the curvature $\kappa\left(\zeta_{0}\right)=\left|\mathbf{y}^{\prime \prime}\left(\zeta_{0}\right)\right|$. Therefore, from the collinearity of $\mathbf{y}^{\prime \prime}\left(\zeta_{0}\right)$ and $\mathbf{l},\left|\varphi^{\prime \prime}\left(\zeta_{0}\right)\right|=\left|\alpha-\alpha^{\prime}\right| \kappa\left(\zeta_{0}\right)$. Finally, the strict convexity of $D$, and the definition of $\varphi(\zeta)$, imply that $\zeta_{0}$ is the unique point of minimum of $\varphi$ on $[0, L]$, and

$$
\frac{\varphi^{\prime \prime}\left(\zeta_{0}\right)}{\left|\varphi^{\prime \prime}\left(\zeta_{0}\right)\right|}=1
$$

Using (23)-(24), expression (21) becomes:

$$
A\left(\alpha^{\prime}, \alpha\right)=-\frac{1 \cdot \alpha}{\sqrt{\left|\alpha-\alpha^{\prime}\right| \kappa\left(\zeta_{0}\right)}} e^{i k\left(\alpha-\alpha^{\prime}\right) \cdot \mathbf{y}\left(\zeta_{0}\right)}\left[1+O\left(\frac{1}{k}\right)\right], \quad k \rightarrow \infty .
$$


At the specular point one has $\mathbf{l} \cdot \alpha^{\prime}=-\mathbf{l} \cdot \alpha$. By the definition $\alpha-\alpha^{\prime}=$ $\mathbf{l}\left|\alpha-\alpha^{\prime}\right|$. Hence $\mathbf{l} \cdot\left(\alpha-\alpha^{\prime}\right)=\left|\alpha-\alpha^{\prime}\right|$ and $2 \mathbf{l} \cdot \alpha=\left|\alpha-\alpha^{\prime}\right|$. These equalities and $d(\mathbf{l})=\mathbf{y}\left(\zeta_{0}\right) \cdot \mathbf{l}$ give

$$
A\left(\alpha^{\prime}, \alpha\right)=-\frac{1}{2} \sqrt{\frac{\left|\alpha-\alpha^{\prime}\right|}{\kappa\left(\zeta_{0}\right)}} e^{i k\left|\alpha-\alpha^{\prime}\right| d(\mathbf{l})}\left[1+O\left(\frac{1}{k}\right)\right], \quad k \rightarrow \infty .
$$

Approximation

$$
A\left(\alpha^{\prime}, \alpha\right) \approx-\frac{1}{2} \sqrt{\frac{\left|\alpha-\alpha^{\prime}\right|}{\kappa\left(\zeta_{0}\right)}} e^{i k\left|\alpha-\alpha^{\prime}\right| d(\mathbf{l})}
$$

can be used for an approximate recovery of the curvature and the support function (modulo $2 \pi / k\left|\alpha-\alpha^{\prime}\right|$ ) of the obstacle. The uncertainty in the support function determination can be remedied by using different combinations of vectors $\alpha$ and $\alpha^{\prime}$.

\section{Numerical results.}

In this numerical experiment the obstacle is the circle

$$
D=\left\{\left(x_{1}, x_{2}\right) \in \mathbb{R}^{2}:\left(x_{1}-6\right)^{2}+\left(x_{2}-2\right)^{2}=1\right\} .
$$

It is reconstructed using the Support Function Method for two frequencies in the resonance region: $k=1.0$, and $k=5.0$. Table 1 shows how well the approximation (27) is satisfied for various pairs of vectors $\alpha$ and $\alpha^{\prime}$ all representing the same vector $\mathbf{l}=(1.0,0.0)$ according to $(22)$. The Table shows the ratios of the approximate Scattering Amplitude $A_{a}\left(\alpha^{\prime}, \alpha\right)$ defined as the right hand side of the equation (27) to the exact Scattering Amplitude $A\left(\alpha^{\prime}, \alpha\right)$. Note, that for a sphere of radius $a$, centered at $\mathbf{x}_{0} \in \mathbb{R}^{2}$, one has

$$
A\left(\alpha^{\prime}, \alpha\right)=-\sqrt{\frac{2}{\pi k}} e^{-i \frac{\pi}{4}} e^{i k\left(\alpha-\alpha^{\prime}\right) \cdot \mathbf{x}_{0}} \sum_{l=-\infty}^{\infty} \frac{J_{l}(k a)}{H_{l}^{(1)}(k a)} e^{i l(\theta-\beta)},
$$

where $\alpha^{\prime}=\mathbf{x} /|\mathbf{x}|=e^{i \theta}$, and $\alpha=e^{i \beta}$. Vectors $\alpha$ and $\alpha^{\prime}$ are defined by their polar angles shown in Table 1.

Table 1 shows that only vectors $\alpha$ close to the vector 1 are suitable for the Scattering Amplitude approximation. This shows the practical importance of the backscattering data. As mentioned at the end of Section 2, any single combination of vectors $\alpha$ and $\alpha^{\prime}$ representing $l$ is not sufficient to uniquely determine the Support Function $d(\mathbf{l})$ from (27) because of the phase uncertainty. However, one can remedy this by using more than one pair of vectors $\alpha$ and $\alpha^{\prime}$ as follows. Let $1 \in S^{1}$ be fixed. Let

$$
R(\mathbf{l})=\left\{\alpha \in S^{1}:|\alpha \cdot \mathbf{l}|>1 / \sqrt{2}\right\} .
$$


Table 1. Ratios of the approximate and the exact Scattering Amplitudes $A_{a}\left(\alpha^{\prime}, \alpha\right) / A\left(\alpha^{\prime}, \alpha\right)$ for $\mathbf{l}=(1.0,0.0)$.

\begin{tabular}{|rrcc|}
\hline$\alpha^{\prime}$ & $\alpha$ & $k=1.0$ & $k=5.0$ \\
\hline$\pi$ & 0 & $0.88473-0.17487 i$ & $0.98859-0.05846 i$ \\
$23 \pi / 24$ & $\pi / 24$ & $0.88272-0.17696 i$ & $0.98739-0.06006 i$ \\
$22 \pi / 24$ & $2 \pi / 24$ & $0.87602-0.18422 i$ & $0.98446-0.06459 i$ \\
$21 \pi / 24$ & $3 \pi / 24$ & $0.86182-0.19927 i$ & $0.97977-0.07432 i$ \\
$20 \pi / 24$ & $4 \pi / 24$ & $0.83290-0.22411 i$ & $0.96701-0.08873 i$ \\
$19 \pi / 24$ & $5 \pi / 24$ & $0.77723-0.25410 i$ & $0.95311-0.10321 i$ \\
$18 \pi / 24$ & $6 \pi / 24$ & $0.68675-0.27130 i$ & $0.92330-0.14195 i$ \\
$17 \pi / 24$ & $7 \pi / 24$ & $0.57311-0.25360 i$ & $0.86457-0.14959 i$ \\
$16 \pi / 24$ & $8 \pi / 24$ & $0.46201-0.19894 i$ & $0.81794-0.22900 i$ \\
$15 \pi / 24$ & $9 \pi / 24$ & $0.36677-0.12600 i$ & $0.61444-0.19014 i$ \\
$14 \pi / 24$ & $10 \pi / 24$ & $0.28169-0.05449 i$ & $0.57681-0.31075 i$ \\
$13 \pi / 24$ & $11 \pi / 24$ & $0.19019+0.00075 i$ & $0.14989-0.09479 i$ \\
$12 \pi / 24$ & $12 \pi / 24$ & $0.00000+0.00000 i$ & $0.00000+0.00000 i$ \\
\hline
\end{tabular}

Define $\Psi: \mathbb{R} \rightarrow \mathbb{R}^{+}$by

$$
\Psi(t)=\left\|A\left(\alpha^{\prime}, \alpha\right)+\frac{1}{2} \sqrt{\frac{\left|\alpha-\alpha^{\prime}\right|}{\kappa\left(\zeta_{0}\right)}} e^{i k\left|\alpha-\alpha^{\prime}\right| t}\right\|_{L^{2}(R(1))}^{2},
$$

where $\alpha^{\prime}=\alpha^{\prime}(\alpha)$ is defined by $\mathbf{l}$ and $\alpha$ according to (22), and the integration is done over $\alpha \in R(\mathbf{l})$.

If the approximation (27) were exact for any $\alpha \in R(\mathbf{l})$, then the value of $\Psi(d(\mathbf{l}))$ would be zero. This justifies the use of the minimizer $t_{0} \in \mathbb{R}$ of the function $\Psi(t)$ as an approximate value of the Support Function $d(\mathbf{l})$. If the Support Function is known for sufficiently many directions $\mathbf{l} \in S^{1}$, the obstacle can be localized using (12) or (16). The results of such a localization for $k=1.0$ together with the original obstacle $D$ is shown on Figure 1. For $k=5.0$ the identified obstacle is not shown, since it is practically the same as $D$. The only a priori assumption on $D$ was that it was located inside the circle of radius 20 with the center in the origin. The Support Function was computed for 16 uniformly distributed in $S^{1}$ vectors 1 .

\section{Conclusions.}

The Support Function Method is an inexpensive algorithm for solution of Inverse Obstacle Scattering problems. The SFM can be used in conjunction 


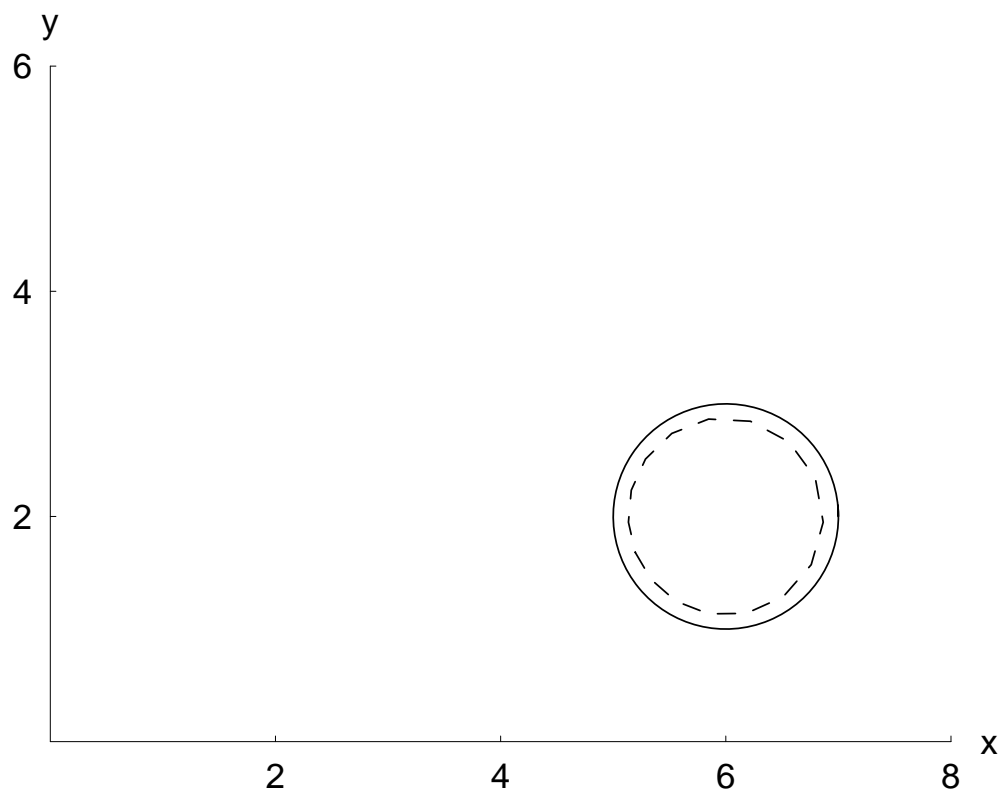

Figure 1. Identified (dotted line), and the original (solid line) obstacle $D$ for $k=1.0$.

with other methods (such as the Modified Rayleigh Conjecture (MRC) method, see $\left.{ }^{1},{ }^{4}\right)$, which can provide a more precise identification, given an approximate location of the obstacle.

\section{References}

1. Gutman S., Ramm A.G. [2002] Numerical Implementation of the MRC Method for Obstacle Scattering Problems, Jour. Phys. A, 35, 8065-8074.

2. Ramm A.G. [1986] Scattering by Obstacles, D. Reidel Publishing, Dordrecht, Holland.

3. Ramm A.G. [1992] Multidimensional Inverse Scattering Problems, Longman/Wiley, New York.

4. Ramm A.G. [2002] Modified Rayleigh conjecture and applications, Jour. Phys. A, 35, L357-361. 